\title{
Extracellular vesicles isolated from dsRNA sprayed barley plants exhibit no growth inhibition or gene silencing in Fusarium graminearum
}

\section{Timo Schlemmer}

Justus Liebig Universitat Giessen https://orcid.org/0000-0003-4034-445X

Richard Lischka

Justus Liebig Universitat Giessen

Dagmar Biedenkopf

Justus Liebig Universitat Giessen

Aline Koch ( $\nabla$ aline.koch@uni-hohenheim.de)

University of Hohenheim https://orcid.org/0000-0003-3536-5344

\section{Short Report}

Keywords: Extracellular vesicles, plant EVs, barley, Fusarium graminearum, RNAi, RNA biopesticides, spray-induced gene silencing, RNAi-based plant protection, dsRNA, siRNA

Posted Date: September 30th, 2021

DOI: https://doi.org/10.21203/rs.3.rs-944619/v1

License: (9) This work is licensed under a Creative Commons Attribution 4.0 International License. Read Full License 
1 Extracellular vesicles isolated from dsRNA sprayed barley plants exhibit no growth inhibition or gene silencing in Fusarium graminearum

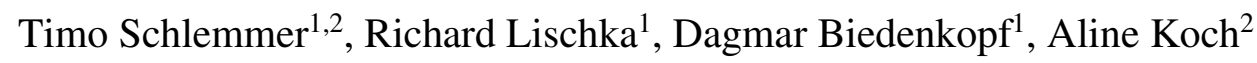

${ }^{1}$ Institute of Phytopathology, Centre for BioSystems, Land Use and Nutrition, Justus Liebig University, Heinrich-Buff-Ring 26, D-35392, Giessen, Germany

${ }^{2}$ Institute of Phytomedicine, University of Hohenheim, Otto-Sander-Strasse 5, D-70599, Stuttgart, Germany

Corresponding author: Aline Koch

Email: aline.koch@uni-hohenheim.de

\section{Abstract}

Incorporating a double-stranded RNA (dsRNA)-expressing transgene into plants or applying dsRNA by spraying it onto plant leaves successfully protects plants against invading pathogens with RNA interference (RNAi). How dsRNAs or siRNAs are transferred between donor host cells and recipient fungal cells is largely unknown It is speculated that plant extracellular vesicles (EVs) function as RNA shuttles between plants and their interacting pathogens. Recently, we found that EVs isolated from HIGS or SIGS plants contained dsRNA-derived siRNAs. In this study, we evaluated whether isolated EVs from RNA-sprayed barley (Hordeum vulgare) plants affected the growth of the phytopathogenic ascomycete Fusarium graminearum $(F g)$. Encouraged by our previous finding that dropping barley-derived EVs on $F g$ cultures caused fungal stress phenotypes, we conducted an in vitro growth experiment in microtiter plates where we co-cultivated $F g$ with plant EVs isolated from dsRNA-sprayed barley leaves. We observed that co-cultivation of $F g$ macroconidia with barley EVs did not affect fungal growth. Furthermore, plant EVs containing SIGS-derived siRNA appeared not to affect $F g$ growth and showed no gene silencing activity on $F g C Y P 51$ genes. We conclude that either the 
amount of spray-derived sRNA was insufficient to induce target gene silencing (SIGS) in $F g$ or $F g$ uptake of plant EVs from liquid cultures was inefficient or impossible.

\section{Keywords}

Extracellular vesicles, plant EVs, barley, Fusarium graminearum, RNAi, RNA biopesticides, spray-induced gene silencing, RNAi-based plant protection, dsRNA, siRNA

\section{Background}

Research on plant extracellular vesicles (EVs) has exploded since the first reports in the 1970s, and the number of scientific studies on them has quintupled over the last decade. Plant EVs attract immense scientific interest due to the anti-inflammatory, anticancer, antioxidative and antisenescence properties of EVs derived or isolated from, e.g., Panax ginseng (Cho et al., 2021), Asparagus cochinchinensis (He, 2021), Aloe vera (Kim et al., 2021), Allium sativum (Özkan et al., 2021), bitter melon (Yang et al., 2021), grapefruit (Stanly et al., 2020), strawberry (Perut et al., 2021), carrot (Kim and Rhee, 2021) and honey (Chen et al., 2021). Their strong bioactivity and biocompatibility together with efficient uptake by human cells have raised the possibility of exploiting them as novel drug delivery vehicles (Bokka et al., 2020; Niu et al., 2021; You et al., 2021). The bioengineering of plant EVs to develop next-generation therapeutic tools in nanomedicine has also inspired plant biotechnologists who continuously seek sustainable and eco-friendly solutions in agriculture and plant protection. Thus, using EVs as natural blueprints may stimulate and further boost the development of nanocarrier-based technologies that facilitate the efficient delivery of CRISPR/Cas components or systems in the future (Alghuthaymi et al., 2021). However, applied plant EV research is a more recent development that has undergone rapid progress in the last three years, with more than 260 studies published ${ }^{1}$.

${ }^{1}$ PubMed (nih.gov) 
Although plant EVs were first described in the apoplast in 1967 (Halperin and Jensen, 1967), it was almost half a century before they were separated from plant apoplastic fluids and then visualized with transmission electron microscopy (TEM) (Regente et al., 2009, 2017; Rutter and Innes, 2017). These pioneering works have laid the foundation for studies demonstrating the pivotal role of EVs in plant-pathogen interactions (Cai et al., 2021; Stotz et al., 2021). For example, plant- or pathogen-derived EVs contribute bidirectionally to this highly specialized interspecies communication through the release of lipids, proteins and small RNAs (sRNAs) that regulate and deregulate defence and offence responses (Cai et al., 2020; Liu et al., 2020; De Palma et al., 2020; Cavaco et al., 2021; Woith et al., 2021). In particular, the identification of plant EV-derived sRNAs furnished strong evidence for their role as shuttles in interspecies communication and mechanistic insights into plant antifungal defence responses (Cai et al., 2018; Baldrich et al., 2019; Roth et al., 2019; Schlemmer et al. 2020; 2021a; 2021b). Conversely, fungal pathogens secrete sRNAs to dampen plant immunity (Weiberg et al., 2013; Kusch et al., 2018; Dunker et al., 2020; Werner et al., 2021). This sRNA-based crosstalk, also known as cross-species RNA interference (RNAi), was first described in 2013, in a paper demonstrating that the fungal pathogen Botrytis cinerea produces sRNAs that mimic plant sRNAs and bind to Arabidopsis AGO1 to antagonistically silence important plant immunity genes (Weiberg et al., 2013). Similar to plant EV-mediated sRNA transport, it is proposed that fungal sRNA delivery is facilitated by EVs (Kwon et al., 2020). To support this hypothesis, EVs isolated from different fungal pathogens, such as Ustilago maydis (Kwon et al., 2021), Zymoseptoria tritici (Hill and Solomon, 2020), Fusarium oxysporum (Bleackley et al., 2020; Garcia-Ceron et al., 2021) and Fusarium graminearum (Fg) (Schlemmer et al. 2020), were described for subsequent RNA content analysis.

In agriculture, RNAi technologies attract immense scientific and political interest as powerful substitutes for conventional chemical pesticides to reach the EU's sustainability goals (Taning et al., 2021). Currently, RNAi-based plant protection relies on two strategies that differ in the 
origin of dsRNA. First, endogenous dsRNA formation mediated by transgene expression is designated as host-induced gene silencing (HIGS). The second strategy is based on exogenous, foliar dsRNA application known as spray-induced gene silencing (SIGS). Notably, the principle of cross-species RNAi was biotechnologically used (HIGS) before its naturally occurring equivalent was discovered (Nowara et al., 2010). We previously demonstrated that a transgenederived CYP3RNA (a dsRNA designed to target CYP51A, CYP51B and CYP51C genes in $F g$ ), as well as foliar application of CYP3RNA, induced CYP51 target gene silencing in Fg. Remarkably, HIGS-or SIGS-mediated FgCYP51 downregulation conferred strong $F g$ disease resistance in Arabidopsis thaliana (HIGS) and Hordeum vulgare (HIGS and SIGS) (Koch et al., 2013, 2016, 2019; Biedenkopf et al., 2020; Höfle et al., 2020).

Recently, we showed that EVs isolated from CYP3RNA-expressing Arabidopsis plants contain CYP3RNA-derived siRNAs (Schlemmer et al 2021a). Notably, subsequent differential digestive treatments of EVs with RNase, protease and a detergent revealed that, compared with that in the apoplastic fluid of Arabidopsis, the amount of intravesicular sRNA was surprisingly low (Schlemmer et al. 2021a). In addition, we observed a loss of CYP3RNA/HIGS-mediated Fg disease resistance in Arabidopsis with a mutated endosomal sorting complex required for transport III (ESCRT-III), which is necessary for proper EV biogenesis (Schlemmer et al. 2021a). Moreover, ESCRT-III mutants were impaired in EV production and no CYP3RNAderived siRNAs were detected in either the apoplastic fluid or inside the EVs (Schlemmer et al. 2021a). EVs isolated from CYP3RNA-sprayed barley plants revealed CYP3RNA-derived siRNAs; however, their abundance was even lower compared with Arabidopsis EVs (Schlemmer et al 2021b). This difference might be due to the various dsRNA origins in HIGS and SIGS approaches, whereby SIGS-induced RNAs must be taken up by plant cells before being packed into plant EVs (Koch and Wassenegger, 2021). CYP3RNA uptake into plant cells and its systemic spread via the phloem have been previously reported as well as its apoplastic 
transport in the xylem (Koch et al., 2016; Biedenkopf et al., 2020). However, whether EVs are required for the delivery of exogenously applied dsRNA to induce SIGS in $F g$ remains unclear. To address this question, we assessed whether EVs isolated from SIGS plants can induce FgCYP51 target gene silencing and $F g$ growth inhibition. We performed in vitro treatments of $F g$ with EVs isolated from CYP3RNA sprayed barley plants. Remarkably, we found no effects on $F g C Y P 51$ expression or $F g$ growth, further underlining the importance of clarifying whether EV-mediated sRNA transport is required during SIGS-barley-Fg interaction.

\section{Results}

To test the possibility of plant EV uptake by Fg in vitro, we isolated EVs from control(Tris-EDTA buffer) and CYP3RNA-sprayed barley leaves using a modified protocol as described (Rutter and Innes, 2017; Schlemmer et al 2020). In our recent studies, we observed that state-of-the-art EV purification from apoplastic fluids leads to impure EV isolates containing additional co-purified apoplastic substances (Schlemmer et al., 2021a). This finding aligns with recent debates discussing the pitfalls of and the standardization needs in plant EV research, e.g., the contamination risks of different plant EV separation and characterization methods (Rutter and Innes, 2020; Mammadova et al., 2021; Pinedo et al., 2021). To avoid such pitfalls, we performed a stringent digestive treatment of EV isolates to degrade extravesicular proteins and RNAs before in vitro treatment of $F g$ with plant EVs. Each EV isolate was derived from 80 barley leaves and EVs were ultimately resuspended in $190 \mu 1 \mathrm{PBS}$. We reserved $40 \mu 1$ for quality control measurements, TEM and nanoparticle trafficking analysis (NTA). The remaining resuspension was divided into three equal fractions (Fig. 1). To degrade extravesicular proteins, RNAs and ribonucleoprotein complexes, one fraction of EV isolates were treated with proteinase $\mathrm{K}$ and $\mathrm{RNase} \mathrm{A}(\mathrm{PK}+\mathrm{RA})$. In addition to $\mathrm{PK}+\mathrm{RA}$, the next fraction was treated with triton $\mathrm{X}(\mathrm{TX}+\mathrm{PK}+\mathrm{RA})$ to break up EVs and degrade extravesicular and intravesicular proteins, RNAs and ribonucleoprotein complexes (Fig. 1). One fraction remained 
untreated to evaluate whether the observed effects resulted from EVs or co-purified apoplastic fluid proteins or RNAs. Finally, EVs were co-inoculated with $F g$ macroconidia and fungal growth was determined, after 20 hours of pre-incubation, by optical density measurements (OD) every 20 minutes for a further 24 hours.

To assess whether the effects depended on the investigated volumes, we used two different amounts of resuspended EV solution. We tested untreated EVs isolated from TE- or CYP3RNA-sprayed barley leaves and EVs treated with PK+RA and TX+PK+RA. We added 5 $\mu 1$ or $10 \mu 1$ of each EV fraction to $F g$ macroconidia. Regardless of whether EVs were derived from CYP3RNA- or TE-sprayed barley leaves or how EVs were treated after purification, no differences in $\mathrm{Fg}$ growth were observed between treatment volumes (Fig. 2). At the beginning of the measurement period, 23 hours post-inoculation (hpi), all samples showed an OD value around 0.5. At $42 \mathrm{hpi}$, the OD had increased for untreated EVs and PK+RA-treated EVs up to 0.9-1.1, while the OD of TX+PK+RA-treated EVs only rose to 0.7-0.9.

As we observed no difference in the effect on fungal growth induced by different EV volumes, we next assessed the effect of EV treatments after EV isolation on $F g$ growth. As a control, we used EV-free PBS, which was also used for EV resuspension after isolation. We compared the fungal growth over the measured time among the different EV treatments and the investigated amount of EVs. Focusing on $F g$ growth co-cultivated with EVs from TE-sprayed barley leaves independent of the applied volume of EVs, we observed that PK+RA-treated EVs promoted $F g$ growth compared with PBS-treated $F g$ cultures (Fig. 3; Fig. 1), possibly triggered by simplified nutrient uptake via the degraded proteins and RNAs the enzymatic treatment created or by the destruction of proteins that usually inhibit $F g$. However, we did not observe growth promotion when $F g$ was fed with untreated EVs. The same observation was made when we focused on EVs from CYP3RNA-sprayed barley leaves, where no difference in the fungal growth was visible when differently prepared EVs were applied to $F g$. Regardless of whether EVs originated from TE- or CYP3RNA-sprayed barley leaves and whether $5 \mu 1$ or $10 \mu 1$ were 
applied, $F g$ co-cultivated with TX+PK+RA-treated EVs was more inhibited than $F g$ cocultivated with PBS, untreated EVs, or PK+RA-treated EVs (Fig. 3). We, therefore, tested the detergents' effects on $F g$. We mixed TX, PK, RA, PK+RA and TX+PK+RA with PBS, incubated them under the same conditions as the plant EVs and tested the mixtures in our growth assay. We observed no difference in the growth behaviour of $F g$ treated with PK, RA or a combination of both (Fig. 4). Notably, TX or TX with PK+RA led to a clear growth reduction compared with the PBS control, indicating a clear effect of TX on fungal growth independent of plant EVs (Fig. 4; Fig. 1). To avoid misinterpreting the effect of TX as the effect of the investigated CYP3RNA, we calculated the relative growth per EV treatment to compare the effects of TE- and CYP3RNA-sprayed EVs. Remarkably, we found no growth inhibition caused by the CYP3RNA spray application independently of how EVs were treated after isolation (Fig. 5). To verify this result and determine whether the unimpaired fungal growth could be explained by a lack of $F g C Y P 51$ gene silencing, we isolated RNA from the $F g$ cultures grown in microtiter plates and performed $\mathrm{FgCYP} 51$ gene expression analysis. Supporting our previous assumption, we found no gene silencing activity in $F g$ after co-cultivation with EVs isolated from CYP3RNA-sprayed barley leaves (Fig. 6).

\section{Discussion}

The more than 50 studies that demonstrate RNAi-based control of fungal pathogens with an average plant disease resistance of about $60 \%$ (Koch and Wassenegger, 2021) reflect the enormous potential of RNAi technologies to meet the socio-political demand to halve the use of chemical pesticides by 2030 (European Commission, 2021). However, our mechanistic knowledge of HIGS and SIGS is still incomplete, although researchers hope to transition testing from the lab to the field soon (Rank and Koch 2021). Towards this goal, unravelling the routes by which dsRNAs and siRNAs are delivered into fungal cells is key to further improve cellular uptake and systemic distribution and therefore increase the stability and efficacy of sprayed 
RNA biopesticides. RNA uptake and transport essentially serve as effective RNA protection, preventing RNA degradation. Besides RNA stabilization with RNA ribonucleoprotein complexes or lipoproteins, EVs encapsulate RNAs (Lasser et al 2011) thus sheltering them from RNases or degradation in general during short (cell-to-cell) or long-distance (systemic) movement (Valadi et al 2007, Hunter et al 2008). Previously, we found that barley EVs led to stress-related discolouration of $F g$ colonies (Schlemmer et al., 2020) and that CYP3RNAsprayed barley leaves, which confer $F g$ disease resistance (Koch et al., 2016) contained CYP3RNA-derived sRNAs (Schlemmer et al., 2021b). However, as the amount of sprayderived sRNA in barley EVs was low, questions about their role and relevance in SIGS-barley$F g$ interaction arise. To assess this further, we treated $F g$ with EVs isolated from sprayed barley plants in vitro. The impurity of plant EV isolates raised concerns about the reliability of findings and their interpretation (Rutter and Innes, 2020; Mammadova et al., 2021), thus, we performed rigorous digestive treatments of $\mathrm{EV}$ isolates before $\mathrm{Fg}$ in vitro testing. Encouraged by our previous finding that drop inoculation of barley $\mathrm{EVs}$ on $\mathrm{Fg}$ cultures grown on solid agar plates caused an increase in purple pigmentation, indicative of the stress-induced premature formation of fruiting bodies (Schlemmer et al., 2020), we expected to observe the effects of barley EVs on $\mathrm{Fg}$ in liquid in vitro cultures. Interestingly, another recent study demonstrated the antifungal activity of EVs derived from root exudates of tomato plants against Fusarium oxysporum, Botrytis cinerea and Alternaria alternata (De Palma et al., 2020) underlining the validity of in vitro EV-fungal spore interaction tests. Surprisingly, we found that neither wild-type barley EVs nor EVs isolated from CYP3RNA-sprayed barley leaves affected $F g$ growth (Fig. 5). In addition, different EV volumes ( $5 \mu 1$ or $10 \mu \mathrm{lEV}$ suspension) did not affect fungal growth (Fig. 2). In contrast, on solid agar plates, $40 \mu \mathrm{l}$ of EV solution derived from 80 barley leaves was drop-inoculated onto $F g$, suggesting that the tested volumes of $5 \mu 1$ and $10 \mu 1$ might be too low. We also did not observe a CYP3RNA-dependent effect on $F g$ growth (Fig. 5). Based on these results, we hypothesized two possibilities: first, $F g$ is unable to take up EVs in vitro, and second, 
the amount of spray-derived sRNA in EVs is insufficient to induce SIGS. To test the second possibility, we performed $F g C Y P 51$ gene expression analysis on $F g$ cultures after EV treatment, which is a more sensitive way to test CYP3RNA effects on $F g$ than determining the OD of liquid fungal cultures.

Consistent with our results finding no $F g$ growth inhibition, we measured no genesilencing activity in $F g$ co-cultivated with EVs from CYP3RNA barley leaves (Fig. 6). However, this could still be explained by the inability of $F g$ to take up plant EVs in vitro. However, this could be still due to the inability of $F g$ to take up plant EVs in vitro. Notably, plant-derived EVs were shown to contain stress response proteins and lipids (Rutter and Innes, 2017; De Palma et al., 2020; Liu et al., 2020; Cavaco et al., 2021; Schlemmer et al., 2021a) and exhibit antifungal activity (Schlemmer et al. 2020; De Palma et al., 2020). Given this, it is surprising that we found no inhibitory effects of barley-derived EVs. This raises the question of whether EVs or EV content are stable in liquid media, able to overcome the membrane or cellular barriers of $F g$ and able to reach a defined threshold to activate the distinct RNAi machinery in $F g$. While another study convincingly demonstrated sunflower-derived EV uptake by the ascomycete Sclerotinia sclerotiorum through reduced hyphae growth and spore germination (Regente et al., 2017), whether this holds true for other fungi or other pathosystems remains unknown. Notably, the latest studies demonstrated in vitro uptake of plant-derived (ginger, grapefruit, pineapple and paprika) EVs in human and rat cells (Garaeva et al., 2021; Ito et al., 2021; Man et al., 2021), which is of great scientific interest due to their therapeutic potential in nanomedicine (Alfieri et al., 2021). Studies on CYP3RNA-expressing Arabidopsis (HIGS) plants revealed a loss of CYP3RNA-mediated $F g$ resistance in ESCRT-III mutants (Schlemmer et al., 2021a). Additionally, EV purification from these mutants revealed no or aberrant EVs with no CYP3RNA-derived sRNA, indicating the potential role of EVs in HIGSArabidopsis-Fg interaction. However, HIGS in Arabidopsis and SIGS in barley are not mechanistically comparable. 
In summary, we found no $F g$ growth inhibition after treatment of $F g$ in vitro cultures

with CYP3RNA-spray-derived barley EVs. Subsequently, we found no $F g C Y P 51$ target gene silencing, raising the question of whether $F g$ is unable to take up EVs from liquid culture or whether EV-contained CYP3RNA-spray-derived siRNAs are physiologically inactive. However, further research is required to differentiate between the possibility of improper EV uptake and the possibility that the amount of spray-derived sRNA was insufficient to induce FgCYP51 gene silencing (SIGS) by elucidating the role and relevance of EVs for SIGS.

\section{Conclusion}

RNA biopesticides represent a powerful alternative to chemical pesticides. To make future field applications reliable and realistic for agriculture, we require mechanistic knowledge of RNA uptake and interspecies (plant-fungus) sRNA transfer. Identification and characterization of plant and fungal EV content, as well as the mechanisms of loading and release, have begun (He et al., 2021; Woith et al., 2021) but remain limited, unless required exploit EVs as bioagents to confer disease resistance in a more natural context. Importantly, fungal uptake of plant-derived EVs may offer potential routes to cure fungal diseases in humans, based on the emerging evidence that plant-derived EVs exhibit great potential for human health applications (Alfieri et al., 2021). We have just begun to understand the enormous potential underlying natural compounds and delivery routes or compartments as we seek sustainable, biocompatible and biodegradable alternatives to conventional treatments in agriculture as well as medicine. 


\section{Differential EV treatments}

253 EVs of TE- and CYP3RNA-sprayed barley leaves were isolated as described in Schlemmer et al. (2021b). EV isolation was performed in three technical replicates. Each isolation included 80 barley leaves per spray application. EVs were resuspended in $190 \mu 1 \mathrm{PBS}\left(8 \mathrm{mM} \mathrm{NaH}_{2} \mathrm{PO}_{4}\right.$, $150 \mathrm{mM} \mathrm{NaCl}, 3 \mathrm{mM} \mathrm{KCl}$ and $\left.2 \mathrm{mM} \mathrm{KH}_{2} \mathrm{PO}_{4} ; \mathrm{pH} 7.4\right)$ and subdivided into three groups after isolation. The first group was untreated and served as a positive control (Tab. 1). The second group was treated with proteinase $\mathrm{K}$ and $\mathrm{RNase} A(\mathrm{PK}+\mathrm{RA})$ and the third group with triton $\mathrm{X}$, proteinase $\mathrm{K}$ and $\mathrm{RNase} A(\mathrm{TX}+\mathrm{PK}+\mathrm{RA})$ (Tab. 1). All groups were incubated for 30 minutes at $37^{\circ} \mathrm{C}$ and then added to Fusarium graminearum $(F g)$ macroconidia. Table1 Components of the digestive EV treatments for eliminating intravesicular and apoplastic co-purified proteins and RNAs.

TE sprayed barley

dsCYP3RNA sprayed barley

\begin{tabular}{lllllll}
\hline group & 1 & 2 & 3 & 1 & 2 & 3 \\
EV solution & $50 \mu \mathrm{l}$ & $50 \mu \mathrm{l}$ & $50 \mu \mathrm{l}$ & $50 \mu \mathrm{l}$ & $50 \mu \mathrm{l}$ & $50 \mu \mathrm{l}$ \\
RNase & - & $1,2 \mu \mathrm{l}$ & $1,2 \mu \mathrm{l}$ & - & $1,2 \mu \mathrm{l}$ & $1,2 \mu \mathrm{l}$ \\
PK & - & $3 \mu \mathrm{l}$ & $3 \mu \mathrm{l}$ & - & $3 \mu \mathrm{l}$ & $3 \mu \mathrm{l}$ \\
Triton X & - & - & $5,8 \mu \mathrm{l}$ & - & - & $5,8 \mu \mathrm{l}$ \\
PBS & $10 \mu \mathrm{l}$ & $5,8 \mu \mathrm{l}$ & - & $10 \mu \mathrm{l}$ & $5,8 \mu \mathrm{l}$ & - \\
\hline total & $60 \mu \mathrm{l}$ & $60 \mu \mathrm{l}$ & $60 \mu \mathrm{l}$ & $60 \mu \mathrm{l}$ & $60 \mu \mathrm{l}$ & $60 \mu \mathrm{l}$
\end{tabular}

Investigated concentrations: Proteinase K (20 ng/ $\mu$ l) (Thermo Fisher Scientific); RNase A (20 ng/ $\mu 1)$ (Thermo Fisher Scientific); $10 \%$ Triton X-100 (Sigma)

\section{Plant EV - Fg co-culture assay}

Plant EV-Fg co-culture assays were performed in transparent 96-well plates with flat bottoms. $1 / 2$ PDB (potato dextrose broth, Formedium) was used as a carbon source. Each well had 5440 macroconidia, $5 \mu 1$ or $10 \mu 1$ treated EV suspension and PBS added (Tab. 2). 96-well plates were pre-incubated on the lab bench for 20 hours before they were put into a plate reader 
(CLARIOstar, BMG Labtech) for another 24-hour incubation at $25^{\circ} \mathrm{C}$ with $60 \mathrm{rpm}$ shaking where optical density (OD600) was measured every 20 minutes. To exclude microbial contamination from EV isolates and misinterpretation of optical density due to this microbial growth, one control (C) contained no macroconidia (C1) (Tab. 3). Hygromycin was added to inhibit microbial growth and allow changes in optical density to be attributed to fungal growth (C2). C3 contained no PBS but rather an additional 0.5 PDB. C4 contained no EVs. C3 and C4 were used to estimate the effect of the PBS on the optical density and growth behaviour of $F g$. As a reference for different $\mathrm{EV}$ treatments, the effects of $\mathrm{EV}$ treatment detergents were determined by incubating EV-free PBS with PK+RA (C5), TX+PK+RA (C6), PK (C7), RA (C8) and TX (C9) and used during the co-culture assay (Tab. 4). PBS was added to compensate for volume differences resulting from differences in the inserted amounts of EVs. The cocultivation was then performed according to the plant EV-Fg cultivation method described in Tab. 5 .

Table 2 Well composition for microtiter well co-cultivation of differentially treated plant EVs with $F g$.

\begin{tabular}{lllllll} 
group & $\mathbf{1}$ & $\mathbf{1}$ & $\mathbf{2}$ & $\mathbf{2}$ & $\mathbf{3}$ & $\mathbf{3}$ \\
\hline investigated vol. & $5 \mu \mathrm{l}$ & $10 \mu \mathrm{l}$ & $5 \mu \mathrm{l}$ & $10 \mu \mathrm{l}$ & $5 \mu \mathrm{l}$ & $10 \mu \mathrm{l}$ \\
$\mathrm{Fg}$ & $20 \mu \mathrm{l}$ & $20 \mu \mathrm{l}$ & $20 \mu \mathrm{l}$ & $20 \mu \mathrm{l}$ & $20 \mu \mathrm{l}$ & $20 \mu \mathrm{l}$ \\
$0,5 \mathrm{PDB}$ & $125 \mu \mathrm{l}$ & $125 \mu \mathrm{l}$ & $125 \mu \mathrm{l}$ & $125 \mu \mathrm{l}$ & $125 \mu \mathrm{l}$ & $125 \mu \mathrm{l}$ \\
PBS & $10 \mu \mathrm{l}$ & $5 \mu \mathrm{l}$ & $10 \mu \mathrm{l}$ & $5 \mu \mathrm{l}$ & $10 \mu \mathrm{l}$ & $5 \mu \mathrm{l}$ \\
\hline total & $160 \mu \mathrm{l}$ & $160 \mu \mathrm{l}$ & $160 \mu \mathrm{l}$ & $160 \mu \mathrm{l}$ & $160 \mu \mathrm{l}$ & $160 \mu \mathrm{l}$
\end{tabular}

Table 3 Overview of tested controls and their well composition.

\begin{tabular}{lllll} 
controls & C1 & C2 & C3 & C4 \\
\hline$F g$ & & $20 \mu \mathrm{l}$ & $20 \mu \mathrm{l}$ & $20 \mu \mathrm{l}$ \\
$0,5 \mathrm{PDB}$ & $160 \mu \mathrm{l}$ & $125 \mu \mathrm{l}$ & $140 \mu \mathrm{l}$ & $125 \mu \mathrm{l}$
\end{tabular}


PBS

$15 \mu 1$

Hygromycin $\quad 15 \mu 1$

\begin{tabular}{lllll}
\hline total & $160 \mu \mathrm{l}$ & $160 \mu \mathrm{l}$ & $160 \mu \mathrm{l}$ & $160 \mu \mathrm{l}$
\end{tabular}

Table 4 Components of the digestive EV treatments to measure the effects of treatment reagents on fungal growth.

\begin{tabular}{llllll} 
controls & C5 & C6 & C7 & C8 & C9 \\
\hline PBS & $55,8 \mu 1$ & $50 \mu 1$ & $57 \mu l$ & $58,8 \mu 1$ & $54,2 \mu 1$ \\
RNase & $1,2 \mu 1$ & $1,2 \mu 1$ & & $1,2 \mu 1$ & \\
PK & $3 \mu 1$ & $3 \mu 1$ & $3 \mu 1$ & & \\
Triton X & & $5,8 \mu 1$ & & & $5,8 \mu 1$ \\
\hline total & $60 \mu 1$ & $60 \mu 1$ & $60 \mu 1$ & $60 \mu 1$ & $60 \mu 1$
\end{tabular}

290

Table 5 Well composition for microtiter well co-cultivation of EV-free detergent reagents to estimate treatment-dependent effects.

$\begin{array}{llllllllllll}\text { controls } & \mathrm{C5} & \mathrm{C} 5 & \mathrm{C6} & \mathrm{C6} & \mathrm{C7} & \mathrm{C} 7 & \mathrm{C8} & \mathrm{C8} & \mathrm{C} 9 & \mathrm{C9}\end{array}$

\begin{tabular}{|c|c|c|c|c|c|c|c|c|c|c|}
\hline invest. vol. & $5 \mu 1$ & $10 \mu \mathrm{l}$ & $5 \mu 1$ & $10 \mu \mathrm{l}$ & $5 \mu 1$ & $10 \mu 1$ & $5 \mu 1$ & $10 \mu \mathrm{l}$ & $5 \mu 1$ & $10 \mu \mathrm{l}$ \\
\hline $\mathrm{Fg}$ & $20 \mu 1$ & $20 \mu \mathrm{l}$ & $20 \mu 1$ & $20 \mu 1$ & $20 \mu 1$ & $20 \mu 1$ & $20 \mu \mathrm{l}$ & $20 \mu \mathrm{l}$ & $20 \mu 1$ & $20 \mu \mathrm{l}$ \\
\hline \multirow[t]{2}{*}{ 0,5 PDB } & 125 & 125 & 125 & 125 & 125 & 125 & 125 & 125 & $125 \mu \mathrm{l}$ & 125 \\
\hline & $\mu 1$ & $\mu 1$ & $\mu 1$ & $\mu 1$ & $\mu 1$ & $\mu \mathrm{l}$ & $\mu 1$ & $\mu 1$ & & $\mu 1$ \\
\hline PBS & $10 \mu 1$ & $5 \mu \mathrm{l}$ & $10 \mu \mathrm{l}$ & $5 \mu 1$ & $10 \mu \mathrm{l}$ & $5 \mu \mathrm{l}$ & $10 \mu \mathrm{l}$ & $5 \mu 1$ & $10 \mu \mathrm{l}$ & $5 \mu \mathrm{l}$ \\
\hline \multirow[t]{2}{*}{ total } & 160 & 160 & 160 & 160 & 160 & 160 & 160 & 160 & $160 \mu \mathrm{l}$ & 160 \\
\hline & $\mu 1$ & $\mu 1$ & $\mu 1$ & $\mu 1$ & $\mu 1$ & $\mu l$ & $\mu 1$ & $\mu 1$ & & $\mu 1$ \\
\hline
\end{tabular}

\section{FgCYP51 gene silencing analysis}

295 Technical replicates per plate were collected after $44 \mathrm{~h}$ of incubation. RNA extraction, cDNA synthesis and qRT-PCR for transcript analysis of $F g C Y P 51 \mathrm{~A}$ and $F g C Y P 51 C$ were performed as described (Koch et al., 2013, 2016). 
Fig. 1 Schematic overview above the investigated EV treatments and their potential effect on EV and EVs cargo. Fraction one (1) contains untreated EVs from mock or CYP3RNA sprayed barley leaves and cause average fungal growth. EVs of fraction two (2) were treated with proteinase $\mathrm{K}(\mathbf{P K})$ and RNase A (RA) to degrade extravesicular ribonuclear complexes. In fraction three (3) EVs were broken up by triton $\mathrm{x}(\mathbf{T X})$ treatment and cargo was degraded by PK and RA treatment.

Fig. $25 \mu \mathrm{l}$ (light blue cross) and $10 \mu \mathrm{l}$ (grey triangle) of purified EVs from control (Tris-EDTA) and CYP3RNA-sprayed barley leaves were added to $F g$ liquid culture. Growth was determined by optical density measurements between 23 and 42 hpi.

Fig. 3 Purified barley EVs were differentially treated with RNase A and Protease K (yellow square) or Triton-X 100, RNase A and Protease K (green rhombus) after isolation and coinoculated with $F g$. Additionally, untreated (orange circle) and EV-free PBS (brown cross) were co-inoculated as positive and negative controls.

Fig. 4 The effects of investigated enzymes and detergents were evaluated by co-cultivating without barley EVs. $5 \mu \mathrm{l}$ and $10 \mu \mathrm{l}$ were added per enzyme, detergent or combination of both. PBS (negative control: EV-free and enzyme or detergent free; red line) is shown as a reference.

Fig. 5 The relative growth was calculated from co-culture assays with differently treated barley EVs compared with the EV-free cultivation using the enzymes and detergents used for EV treatment. Control (TE: Tris-EDTA): circle; CYP3RNA: triangle.

Fig. 642 hpi of EV-Fg co-cultivation, $F g$ suspension was harvested and technical triplicates for each well were combined before RNA isolation. Transcriptional analyses were performed and $F g C Y P 51 A$ and $F g C Y P 51 C$ expression was calculated with the Delta Delta CT method using the elongation factor $1 \alpha$ as the reference gene. Relative quantification was determined 
for the equivalently co-incubated $F g$ cultures with EVs derived from control-sprayed and equally treated EV fractions.

\section{Funding}

This work was supported by the Deutsche Forschungsgemeinschaft, Research Training Group (RTG) 2355 (project number 325443116) to A.K. and T.S.

\section{Acknowledgements}

We thank Christina Birkenstock for plant cultivation. We thank Georg Petschenka for providing access to the plate reader and Anja Betz for technical support during optical density measurements.

\section{Author contributions}

"Conceptualization, A.K. and T.S.; Methodology, T.S., R.L and D.B; Software, T.S. and R.L; Validation, A.K., T.S. and R.L; Formal Analysis, T.S., R.L and D.B; Investigation, T.S. and R.L; Data Curation, T.S. and R.L; Writing - Original Draft Preparation, A.K. and T.S.; Writing - Review \& Editing, A.K.; Visualization, T.S. and R.L.; Supervision, A.K.; Project Administration, A.K.; Funding Acquisition, A.K.”

\section{Data Availability Statement}

All relevant data is contained within the article. The original contributions presented in the study are included in the article material, further inquiries can be directed to the corresponding author. 
The authors declare no conflict of interest. The authors declare no competing financial interests.

\section{References}

Alfieri, Mariaevelina; Leone, Antonietta; Ambrosone, Alfredo (2021): Plant-Derived Nano and Microvesicles for Human Health and Therapeutic Potential in Nanomedicine. In: Pharmaceutics 13 (4). DOI: 10.3390/pharmaceutics13040498.

Alghuthaymi, Mousa A.; Ahmad, Aftab; Khan, Zulqurnain; Khan, Sultan Habibullah; Ahmed, Farah K.; Faiz, Sajid et al. (2021): Exosome/Liposome-like Nanoparticles: New Carriers for CRISPR Genome Editing in Plants. In: International journal of molecular sciences 22 (14). DOI: $10.3390 / \mathrm{ijms} 22147456$.

Baldrich, Patricia; Rutter, Brian D.; Karimi, Hana Zand; Podicheti, Ram; Meyers, Blake C.; Innes, Roger W. (2019): Plant Extracellular Vesicles Contain Diverse Small RNA Species and Are Enriched in 10- to 17-Nucleotide "Tiny" RNAs. In: The Plant cell 31 (2), S. 315-324. DOI: 10.1105/tpc.18.00872.

Biedenkopf, D.; Will, T.; Knauer, T.; Jelonek, L.; Furch, Alexandra Charlotte Ursula; Busche, T.; Koch, A. (2020): Systemic spreading of exogenous applied RNA biopesticides in the crop plant Hordeum vulgare. In: ExRNA 2 (1). DOI: 10.1186/s41544-020-00052-3.

Bleackley, Mark R.; Samuel, Monisha; Garcia-Ceron, Donovan; McKenna, James A.; Lowe, Rohan G. T.; Pathan, Mohashin et al. (2019): Extracellular Vesicles From the Cotton Pathogen Fusarium oxysporum f. sp. vasinfectum Induce a Phytotoxic Response in Plants. In: Frontiers in plant science 10, S. 1610. DOI: 10.3389/fpls.2019.01610.

Bokka, Ramesh; Ramos, Anna Paulina; Fiume, Immacolata; Manno, Mauro; Raccosta, Samuele; Turiák, Lilla et al. (2020): Biomanufacturing of Tomato-Derived Nanovesicles. In: Foods (Basel, Switzerland) 9 (12). DOI: 10.3390/foods9121852. 
Cai, Qiang; He, Baoye; Wang, Shumei; Fletcher, Stephen; Niu, Dongdong; Mitter, Neena et al. (2021): Message in a Bubble: Shuttling Small RNAs and Proteins Between Cells and Interacting Organisms Using Extracellular Vesicles. In: Annual review of plant biology 72, S. 497-524. DOI: 10.1146/annurev-arplant-081720-010616.

Cai, Qiang; He, Baoye; Weiberg, Arne; Buck, Amy H.; Jin, Hailing (2019): Small RNAs and extracellular vesicles: New mechanisms of cross-species communication and innovative tools for disease control. In: PLoS pathogens 15 (12), e1008090. DOI: 10.1371/journal.ppat.1008090.

Cai, Qiang; Qiao, Lulu; Wang, Ming; He, Baoye; Lin, Feng-Mao; Palmquist, Jared et al. (2018a): Plants send small RNAs in extracellular vesicles to fungal pathogen to silence virulence genes. In: Science (New York, N.Y.) 360 (6393), S. 1126-1129. DOI: 10.1126/science.aar4142.

Cai, Qiang; Qiao, Lulu; Wang, Ming; He, Baoye; Lin, Feng-Mao; Palmquist, Jared et al. (2018b): Plants send small RNAs in extracellular vesicles to fungal pathogen to silence virulence genes. In: Science 360 (6393), S. 1126-1129. DOI: 10.1126/science.aar4142.

Cavaco, Ana Rita; Matos, Ana Rita; Figueiredo, Andreia (2021): Speaking the language of lipids: the cross-talk between plants and pathogens in defence and disease. In: Cell. Mol. Life Sci. 78 (9), S. 4399-4415. DOI: 10.1007/s00018-021-03791-0.

Chen, Xingyi; Liu, Baolong; Li, Xingzhi; An, Thuy T.; Zhou, You; Li, Gang et al. (2021): Identification of anti-inflammatory vesicle-like nanoparticles in honey. In: Journal of extracellular vesicles 10 (4), e12069. DOI: 10.1002/jev2.12069.

Cho, Eun-Gyung; Choi, Suh-Yeon; Kim, Hyoseon; Choi, Eun-Jeong; Lee, Eun-Jeong; Park, Phil-Jun et al. (2021): Panax ginseng-Derived Extracellular Vesicles Facilitate Anti-Senescence Effects in Human Skin Cells: An Eco-Friendly and Sustainable Way to Use Ginseng Substances. In: Cells 10 (3). DOI: 10.3390/cells10030486.

De Palma, Monica; Ambrosone, Alfredo; Leone, Antonietta; Del Gaudio, Pasquale; Ruocco, Michelina; Turiák, Lilla et al. (2020): Plant Roots Release Small Extracellular Vesicles with Antifungal Activity. In: Plants (Basel, Switzerland) 9 (12), S. 1777.Dunker, Florian; Trutzenberg, Adriana; Rothenpieler, Jan S.; Kuhn, Sarah; Pröls, Reinhard; Schreiber, Tom et al. (2020): Oomycete small RNAs bind to the plant RNA-induced silencing complex for virulence. In: eLife 9. DOI: 10.7554/eLife.56096. 
402

403

404

405

406

407

408

409

410

411

412

413

414

415

416

417

418

419

420

421

422

423

424

425

426

427

428

429

430

431

Garaeva, Luiza; Kamyshinsky, Roman; Kil, Yury; Varfolomeeva, Elena; Verlov, Nikolai; Komarova, Elena et al. (2021): Delivery of functional exogenous proteins by plant-derived vesicles to human cells in vitro. In: Scientific reports 11 (1), S. 6489. DOI: 10.1038/s41598021-85833-y.

Garcia-Ceron, Donovan; Dawson, Charlotte S.; Faou, Pierre; Bleackley, Mark R.; Anderson, Marilyn A. (2021): Size-exclusion chromatography allows the isolation of EVs from the filamentous fungal plant pathogen Fusarium oxysporum f. sp. vasinfectum (Fov). In: Proteomics 21 (13-14), e2000240. DOI: 10.1002/pmic.202000240.

Halperin, Walter; Jensen, William A. (1967): Ultrastructural changes during growth and embryogenesis in carrot cell cultures. In: Journal of Ultrastructure Research 18 (3-4), S. 428443. DOI: 10.1016/S0022-5320(67)80128-X.

He, Baoye; Cai, Qiang; Qiao, Lulu; Huang, Chien-Yu; Wang, Shumei; Miao, Weili et al. (2021): RNA-binding proteins contribute to small RNA loading in plant extracellular vesicles. In: Nature plants 7 (3), S. 342-352. DOI: 10.1038/s41477-021-00863-8.

Hill, Erin H.; Solomon, Peter S. (2020): Extracellular vesicles from the apoplastic fungal wheat pathogen Zymoseptoria tritici. In: Fungal biology and biotechnology 7, S. 13. DOI: 10.1186/s40694-020-00103-2.

Höfle, L.; Biedenkopf, D.; Werner, B. T.; Shrestha, A.; Jelonek, L.; Koch, A. (2020): Study on the efficiency of dsRNAs with increasing length in RNA-based silencing of the Fusarium CYP51 genes. In: RNA biology 17 (4), S. 463-473. DOI: 10.1080/15476286.2019.1700033.

Hunter, Melissa Piper; Ismail, Noura; Zhang, Xiaoli; Aguda, Baltazar D.; Lee, Eun Joo; Yu, Lianbo et al. (2008): Detection of microRNA expression in human peripheral blood microvesicles. In: PloS one 3 (11), e3694. DOI: 10.1371/journal.pone.0003694.

Ito, Yuko; Taniguchi, Kohei; Kuranaga, Yuki; Eid, Nabil; Inomata, Yosuke; Lee, Sang-Woong; Uchiyama, Kazuhisa (2021): Uptake of MicroRNAs from Exosome-Like Nanovesicles of Edible Plant Juice by Rat Enterocytes. In: International journal of molecular sciences 22 (7). DOI: $10.3390 /$ ijms22073749.

Kim, Do Kyung; Rhee, Won Jong (2021): Antioxidative Effects of Carrot-Derived Nanovesicles in Cardiomyoblast and Neuroblastoma Cells. In: Pharmaceutics 13 (8). DOI: 10.3390/pharmaceutics13081203. 
Kim, Min Kang; Choi, Young Chan; Cho, Seung Hee; Choi, Ji Suk; Cho, Yong Woo (2021): The Antioxidant Effect of Small Extracellular Vesicles Derived from Aloe vera Peels for Wound Healing. In: Tissue engineering and regenerative medicine 18 (4), S. 561-571. DOI: 10.1007/s13770-021-00367-8.

Koch, Aline; Biedenkopf, Dagmar; Furch, Alexandra; Weber, Lennart; Rossbach, Oliver; Abdellatef, Eltayb et al. (2016): An RNAi-Based Control of Fusarium graminearum Infections Through Spraying of Long dsRNAs Involves a Plant Passage and Is Controlled by the Fungal Silencing Machinery. In: PLoS pathogens 12 (10), e1005901. DOI: 10.1371/journal.ppat.1005901.

Koch, Aline; Höfle, Lisa; Werner, Bernhard Timo; Imani, Jafargholi; Schmidt, Alexandra; Jelonek, Lukas; Kogel, Karl-Heinz (2019): SIGS vs HIGS: a study on the efficacy of two dsRNA delivery strategies to silence Fusarium FgCYP51 genes in infected host and non-host plants. In: Molecular plant pathology 20 (12), S. 1636-1644. DOI: 10.1111/mpp.12866.

Koch, Aline; Kumar, Neelendra; Weber, Lennart; Keller, Harald; Imani, Jafargholi; Kogel, Karl-Heinz (2013): Host-induced gene silencing of cytochrome P450 lanosterol C14 $\alpha$ demethylase-encoding genes confers strong resistance to Fusarium species. In: Proceedings of the National Academy of Sciences of the United States of America 110 (48), S. 19324-19329. DOI: 10.1073/pnas.1306373110.

Koch, Aline; Wassenegger, Michael (2021): Host-induced gene silencing - mechanisms and applications. In: The New phytologist 231 (1), S. 54-59. DOI: 10.1111/nph.17364.

Kusch, Stefan; Frantzeskakis, Lamprinos; Thieron, Hannah; Panstruga, Ralph (2018): Small RNAs from cereal powdery mildew pathogens may target host plant genes. In: Fungal biology 122 (11), S. 1050-1063. DOI: 10.1016/j.funbio.2018.08.008.

Kwon, Seomun; Rupp, Oliver; Brachmann, Andreas; Blum, Christopher Frederik; Kraege, Anton; Goesmann, Alexander; Feldbrügge, Michael (2021): mRNA Inventory of Extracellular Vesicles from Ustilago maydis. In: Journal of fungi (Basel, Switzerland) 7 (7). DOI: 10.3390/jof7070562.

Kwon, Seomun; Tisserant, Constance; Tulinski, Markus; Weiberg, Arne; Feldbrügge, Michael (2020): Inside-out: from endosomes to extracellular vesicles in fungal RNA transport. In: Fungal Biology Reviews 34 (2), S. 89-99. DOI: 10.1016/j.fbr.2020.01.001.

Lässer, Cecilia; Alikhani, Vesta Seyed; Ekström, Karin; Eldh, Maria; Paredes, Patricia Torregrosa; Bossios, Apostolos et al. (2011): Human saliva, plasma and breast milk exosomes 
contain RNA: uptake by macrophages. In: J Transl Med 9 (1), S. 9. DOI: 10.1186/1479-58769-9.

Liu, Ning-Jing; Bao, Jing-Jing; Wang, Ling-Jian; Chen, Xiao-Ya (2020): Arabidopsis leaf extracellular vesicles in wound-induced jasmonate accumulation. In: Plant signaling \& behavior 15 (12), S. 1833142. DOI: 10.1080/15592324.2020.1833142.

Mammadova, Ramila; Fiume, Immacolata; Bokka, Ramesh; Kralj-Iglič, Veronika; Božič, Darja; Kisovec, Matic et al. (2021): Identification of Tomato Infecting Viruses That Co-Isolate with Nanovesicles Using a Combined Proteomics and Electron-Microscopic Approach. In: Nanomaterials (Basel, Switzerland) 11 (8). DOI: 10.3390/nano11081922.

Man, Fulong; Meng, Chen; Liu, Yang; Wang, Yuchen; Zhou, Yun; Ma, Jinqian; Lu, Rong (2021): The Study of Ginger-Derived Extracellular Vesicles as a Natural Nanoscale Drug Carrier and Their Intestinal Absorption in Rats. In: AAPS PharmSciTech 22 (6), S. 206. DOI: 10.1208/s12249-021-02087-7.

Niu, Wenbo; Xiao, Qian; Wang, Xuejiao; Zhu, Junqiao; Li, Jinheng; Liang, Xiaomei et al. (2021): A Biomimetic Drug Delivery System by Integrating Grapefruit Extracellular Vesicles and Doxorubicin-Loaded Heparin-Based Nanoparticles for Glioma Therapy. In: Nano letters 21 (3), S. 1484-1492. DOI: 10.1021/acs.nanolett.0c04753.

Nowara, Daniela; Gay, Alexandra; Lacomme, Christophe; Shaw, Jane; Ridout, Christopher; Douchkov, Dimitar et al. (2010): HIGS: host-induced gene silencing in the obligate biotrophic fungal pathogen Blumeria graminis. In: The Plant cell 22 (9), S. 3130-3141. DOI: 10.1105/tpc. 110.077040 .

Özkan, İrem; Koçak, Polen; Yıldırım, Merve; Ünsal, Naz; Yılmaz, Hazal; Telci, Dilek; Şahin, Fikrettin (2021): Garlic (Allium sativum)-derived SEVs inhibit cancer cell proliferation and induce caspase mediated apoptosis. In: Scientific reports 11 (1), S. 14773. DOI: 10.1038/s41598-021-93876-4.

Perut, Francesca; Roncuzzi, Laura; Avnet, Sofia; Massa, Annamaria; Zini, Nicoletta; Sabbadini, Silvia et al. (2021): Strawberry-Derived Exosome-Like Nanoparticles Prevent Oxidative Stress in Human Mesenchymal Stromal Cells. In: Biomolecules 11 (1). DOI: 10.3390/biom11010087.

Pinedo, Marcela; La Canal, Laura de; Marcos Lousa, Carine de (2021): A call for Rigor and standardization in plant extracellular vesicle research. In: Journal of extracellular vesicles 10 (6), e12048. DOI: 10.1002/jev2.12048. 
Rank AP and Koch A (2021) Lab-to-Field Transition of RNA Spray Applications - How Far are We? Invited Special Issue in Frontiers in Plant Science on the topic 'Advances and Challenges of RNAi Based Technologies for Plants - Volume 2' Front. Plant Sci. in press

Regente, Mariana; Corti-Monzón, Georgina; Maldonado, Ana María; Pinedo, Marcela; Jorrín, Jesús; La Canal, Laura de (2009): Vesicular fractions of sunflower apoplastic fluids are associated with potential exosome marker proteins. In: FEBS letters 583 (20), S. 3363-3366. DOI: 10.1016/j.febslet.2009.09.041.

Regente, Mariana; Pinedo, Marcela; San Clemente, Hélène; Balliau, Thierry; Jamet, Elisabeth; La Canal, Laura de (2017): Plant extracellular vesicles are incorporated by a fungal pathogen and inhibit its growth. In: Journal of experimental botany 68 (20), S. 5485-5495. DOI: $10.1093 / \mathrm{jxb} / \mathrm{erx} 355$.

Roth, Ronelle; Hillmer, Stefan; Funaya, Charlotta; Chiapello, Marco; Schumacher, Karin; Lo Presti, Libera et al. (2019): Arbuscular cell invasion coincides with extracellular vesicles and membrane tubules. In: Nature plants 5 (2), S. 204-211. DOI: 10.1038/s41477-019-0365-4.

Rutter, Brian D.; Innes, Roger W. (2017): Extracellular Vesicles Isolated from the Leaf Apoplast Carry Stress-Response Proteins. In: Plant physiology 173 (1), S. 728-741. DOI: 10.1104/pp.16.01253.

Rutter, Brian D.; Innes, Roger W. (2020): Growing pains: addressing the pitfalls of plant extracellular vesicle research. In: The New phytologist 228 (5), S. 1505-1510. DOI: 10.1111/nph.16725.

Schlemmer, Timo; Barth, Patrick; Weipert, Lisa; Preußer, Christian; Hardt, Martin; Möbus, Anna et al. (2021b): Isolation and Characterization of Barley (Hordeum vulgare) Extracellular Vesicles to Assess Their Role in RNA Spray-Based Crop Protection. In: International journal of molecular sciences 22 (13). DOI: 10.3390/ijms22137212.

Schlemmer, Timo; Lischka, Richard; Koch, Aline (2020): Elucidating the role of extracellular vesicles in the Barley-Fusarium interaction. In: TEV 2 (1), S. 28-35. DOI: 10.47184/tev.2020.01.03.

Schlemmer, Timo; Weipert, Lisa; Barth, Patrick; Werner, Bernhard Timo; Preußer, Christian; Hardt, Martin et al. (2021a): Host-induced gene silencing involves Arabidopsis ESCRT-III pathway for the transfer of dsRNA-derived siRNA. 
Stanly, Christopher; Alfieri, Mariaevelina; Ambrosone, Alfredo; Leone, Antonietta; Fiume, Immacolata; Pocsfalvi, Gabriella (2020): Grapefruit-Derived Micro and Nanovesicles Show Distinct Metabolome Profiles and Anticancer Activities in the A375 Human Melanoma Cell Line. In: Cells 9 (12). DOI: 10.3390/cells9122722.

Stotz, Henrik U.; Brotherton, Dominik; Inal, Jameel (2021): Communication is key: Extracellular vesicles as mediators of infection and defence during host-microbe interactions in animals and plants. In: FEMS microbiology reviews. DOI: 10.1093/femsre/fuab044.

Taning, Clauvis Nji Tizi; Mezzetti, Bruno; Kleter, Gijs; Smagghe, Guy; Baraldi, Elena (2021): Does RNAi-Based Technology Fit within EU Sustainability Goals? In: Trends in biotechnology 39 (7), S. 644-647. DOI: 10.1016/j.tibtech.2020.11.008.

Valadi, Hadi; Ekström, Karin; Bossios, Apostolos; Sjöstrand, Margareta; Lee, James J.; Lötvall, Jan O. (2007): Exosome-mediated transfer of mRNAs and microRNAs is a novel mechanism of genetic exchange between cells. In: Nat Cell Biol 9 (6), S. 654-659. DOI: 10.1038/ncb1596. Weiberg, Arne; Wang, Ming; Lin, Feng-Mao; Zhao, Hongwei; Zhang, Zhihong; Kaloshian, Isgouhi et al. (2013): Fungal small RNAs suppress plant immunity by hijacking host RNA interference pathways. In: Science (New York, N.Y.) 342 (6154), S. 118-123. DOI: 10.1126/science.1239705.

Werner, Bernhard Timo; Koch, Aline; Šečić, Ena; Engelhardt, Jonas; Jelonek, Lukas; Steinbrenner, Jens; Kogel, Karl-Heinz (2021): Fusarium graminearum DICER-like-dependent sRNAs are required for the suppression of host immune genes and full virulence. In: PloS one 16 (8), e0252365. DOI: 10.1371/journal.pone.0252365.

Woith, Eric; Guerriero, Gea; Hausman, Jean-Francois; Renaut, Jenny; Leclercq, Céline C.; Weise, Christoph et al. (2021): Plant Extracellular Vesicles and Nanovesicles: Focus on Secondary Metabolites, Proteins and Lipids with Perspectives on Their Potential and Sources. In: International journal of molecular sciences 22 (7). DOI: 10.3390/ijms22073719.

Yang, Meng; Luo, Qingqiong; Chen, Xu; Chen, Fuxiang (2021): Bitter melon derived extracellular vesicles enhance the therapeutic effects and reduce the drug resistance of 5fluorouracil on oral squamous cell carcinoma. In: Journal of nanobiotechnology 19 (1), S. 259. DOI: 10.1186/s12951-021-00995-1.

You, Jae Young; Kang, Su Jin; Rhee, Won Jong (2021): Isolation of cabbage exosome-like nanovesicles and investigation of their biological activities in human cells. In: Bioactive materials 6 (12), S. 4321-4332. DOI: 10.1016/j.bioactmat.2021.04.023. 
558 Zhang, Lei; He, Fengjun; Gao, Lina; Cong, Minghui; Sun, Juan; Xu, Jialu et al. (2021):

559 Engineering Exosome-Like Nanovesicles Derived from Asparagus cochinchinensis Can Inhibit 560 the Proliferation of Hepatocellular Carcinoma Cells with Better Safety Profile. In: IJN 16, S. 561 1575-1586. DOI: 10.2147/IJN.S293067.

562

563 
1

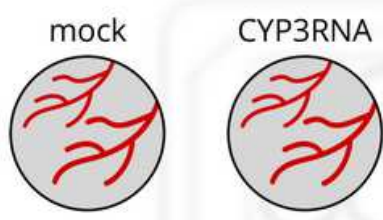

Fusarium Macroconidia

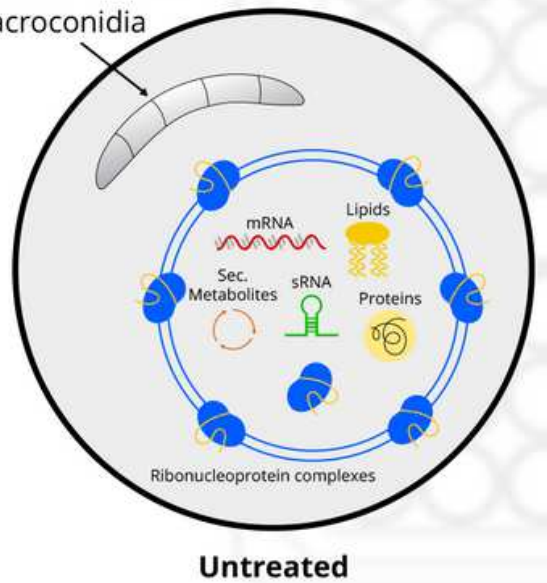

2
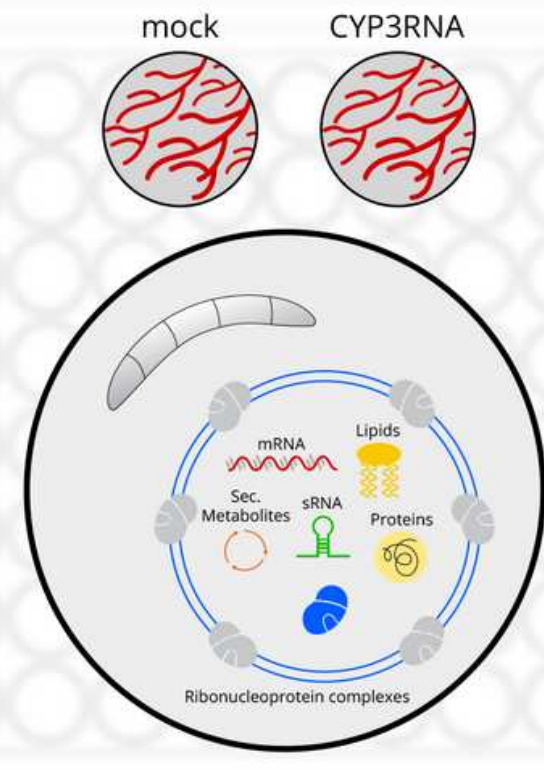

PK+RA
3
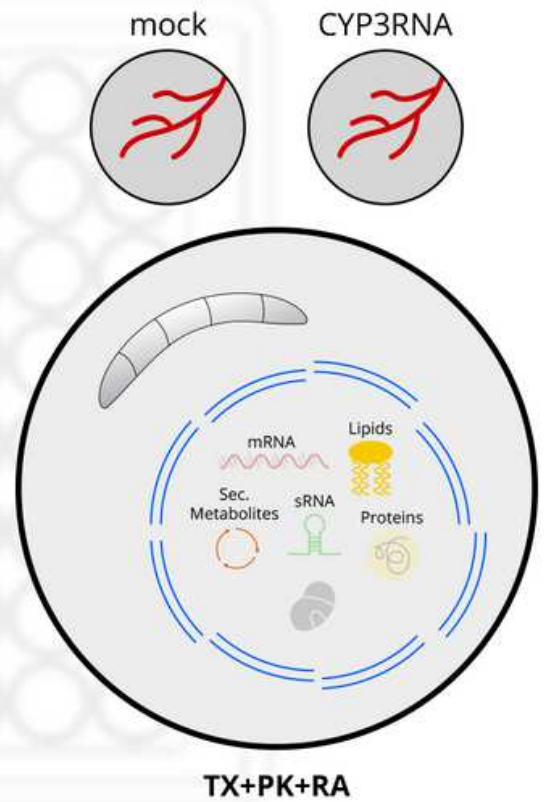

\section{Figure 1}

Schematic overview above the investigated EV treatments and their potential effect on EV and EVs cargo. Fraction one (1) contains untreated EVs from mock or CYP3RNA sprayed barley leaves and cause average fungal growth. EVs of fraction two (2) were treated with proteinase $K(P K)$ and RNase $A(R A)$ to degrade extravesicular ribonuclear complexes. In fraction three (3) EVs were broken up by triton $\mathrm{x}$ (TX) treatment and cargo was degraded by PK and RA treatment. 


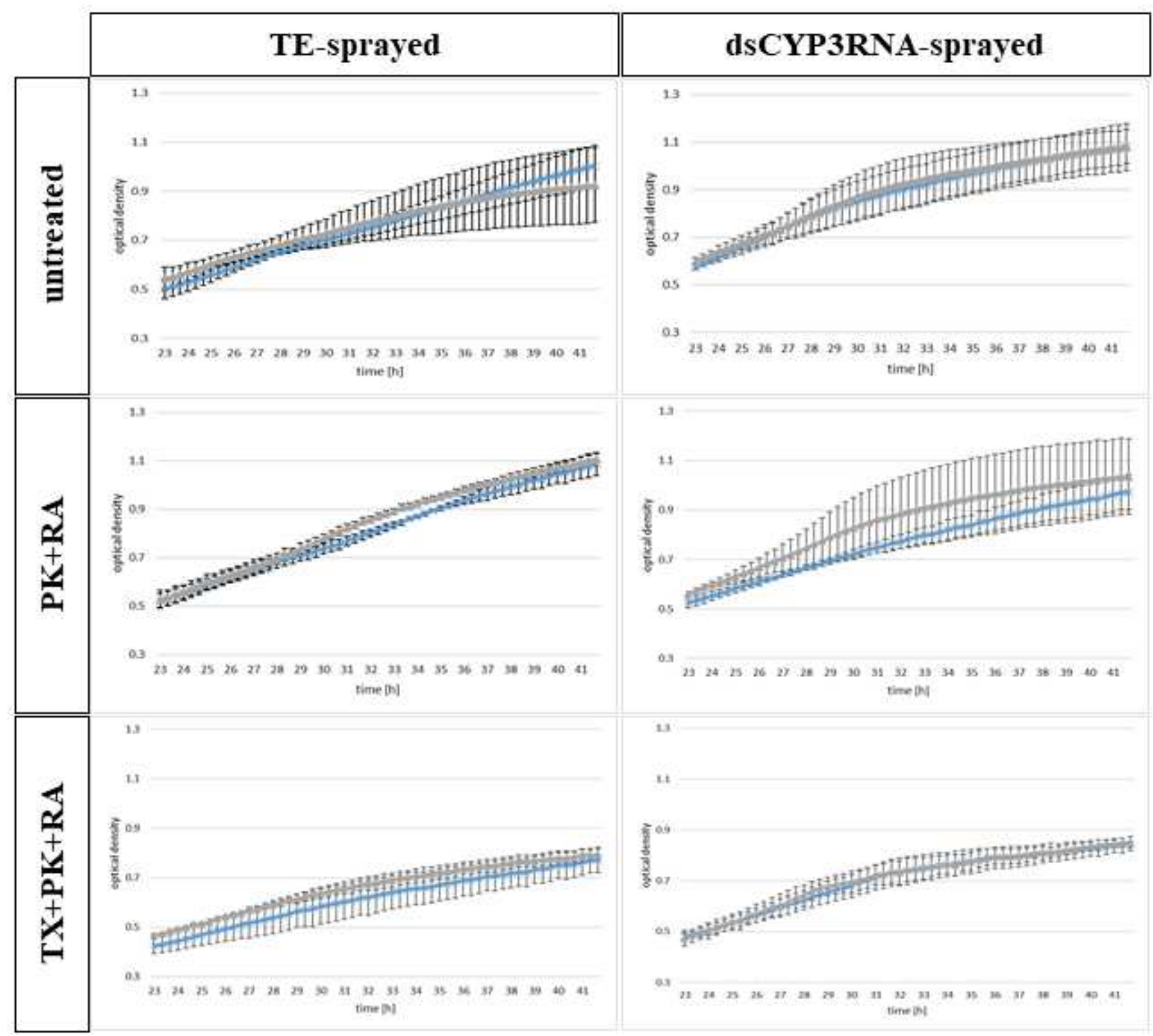

Figure 2

$5 \mu \mathrm{l}$ (light blue cross) and $10 \mu \mathrm{l}$ (grey triangle) of purified EVs from control (Tris-EDTA) and CYP3RNAsprayed barley leaves were added to Fg liquid culture. Growth was determined by optical density measurements between 23 and $42 \mathrm{hpi}$. 


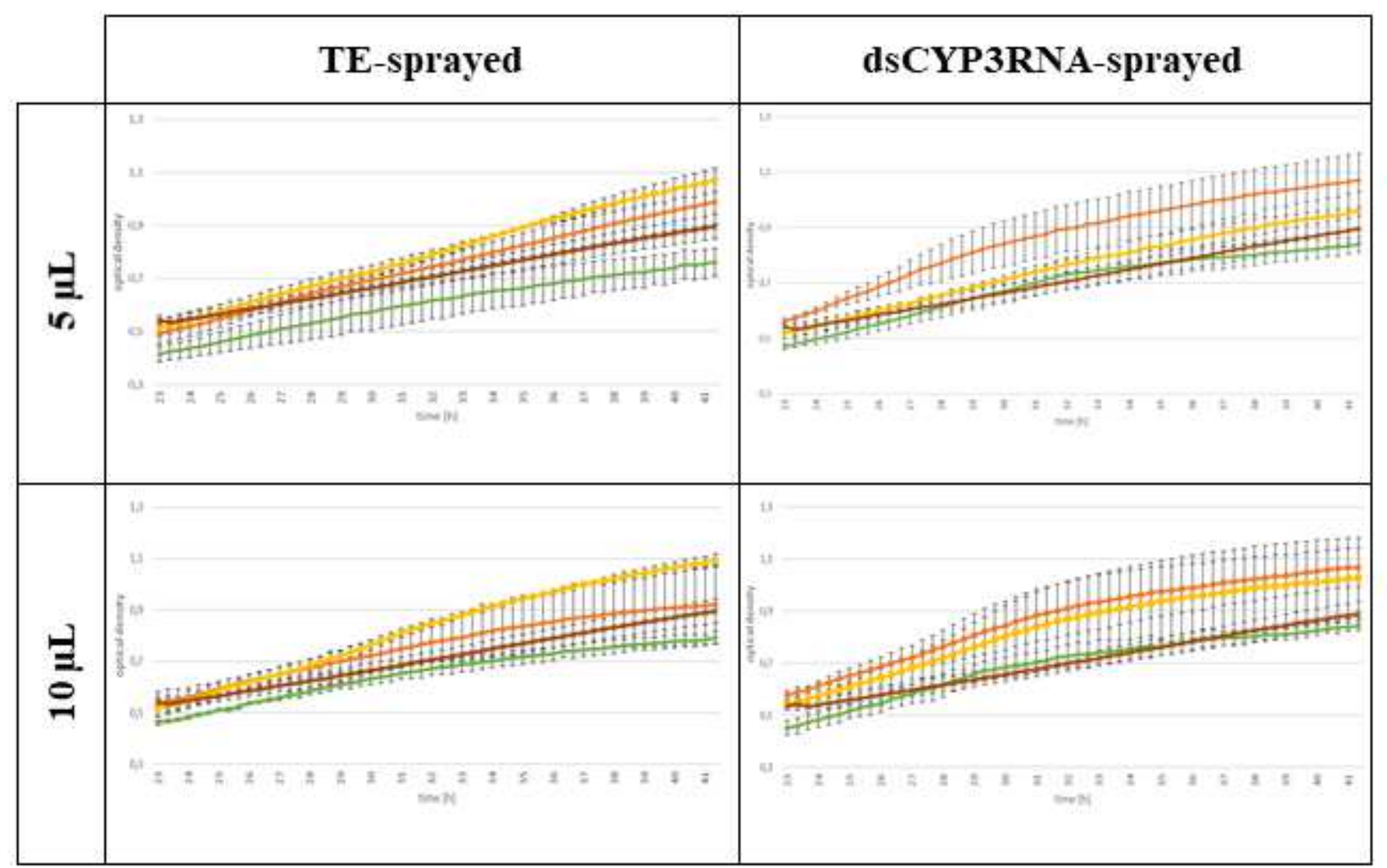

Figure 3

Purified barley EVs were differentially treated with RNase A and Protease K (yellow square) or Triton-X 100, RNase A and Protease K (green rhombus) after isolation and co-inoculated with Fg. Additionally, untreated (orange circle) and EV-free PBS (brown cross) were co-inoculated as positive and negative controls. 

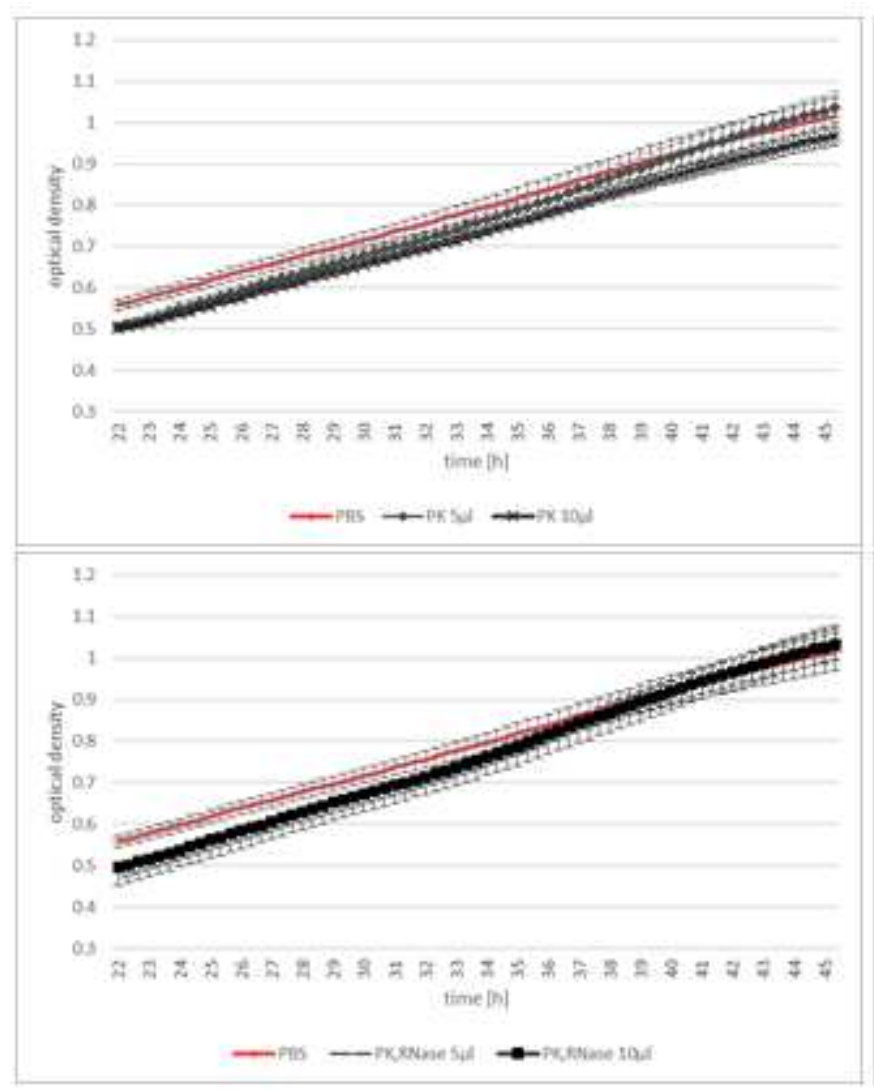

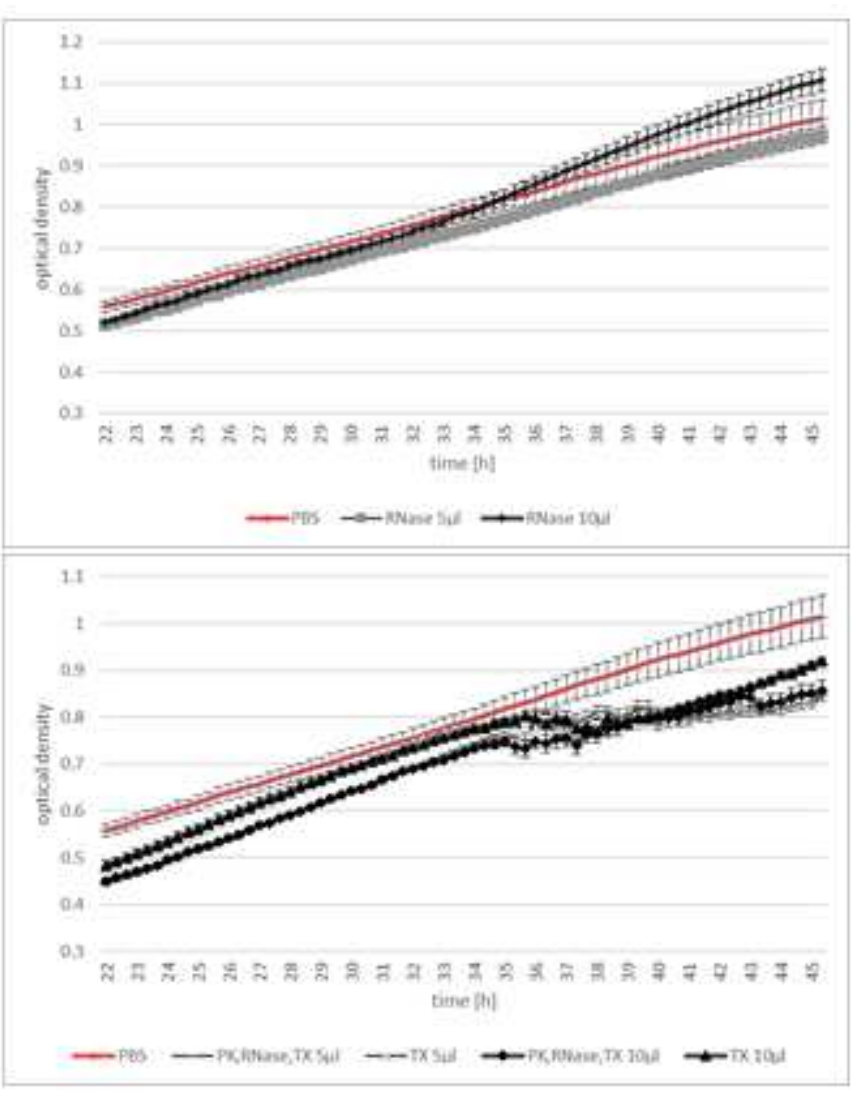

Figure 4

The effects of investigated enzymes and detergents were evaluated by co-cultivating without barley EVs. $5 \mu \mathrm{l}$ and $10 \mu \mathrm{l}$ were added per enzyme, detergent or combination of both. PBS (negative control: EV -free and enzyme or detergent free; red line) is shown as a reference. 

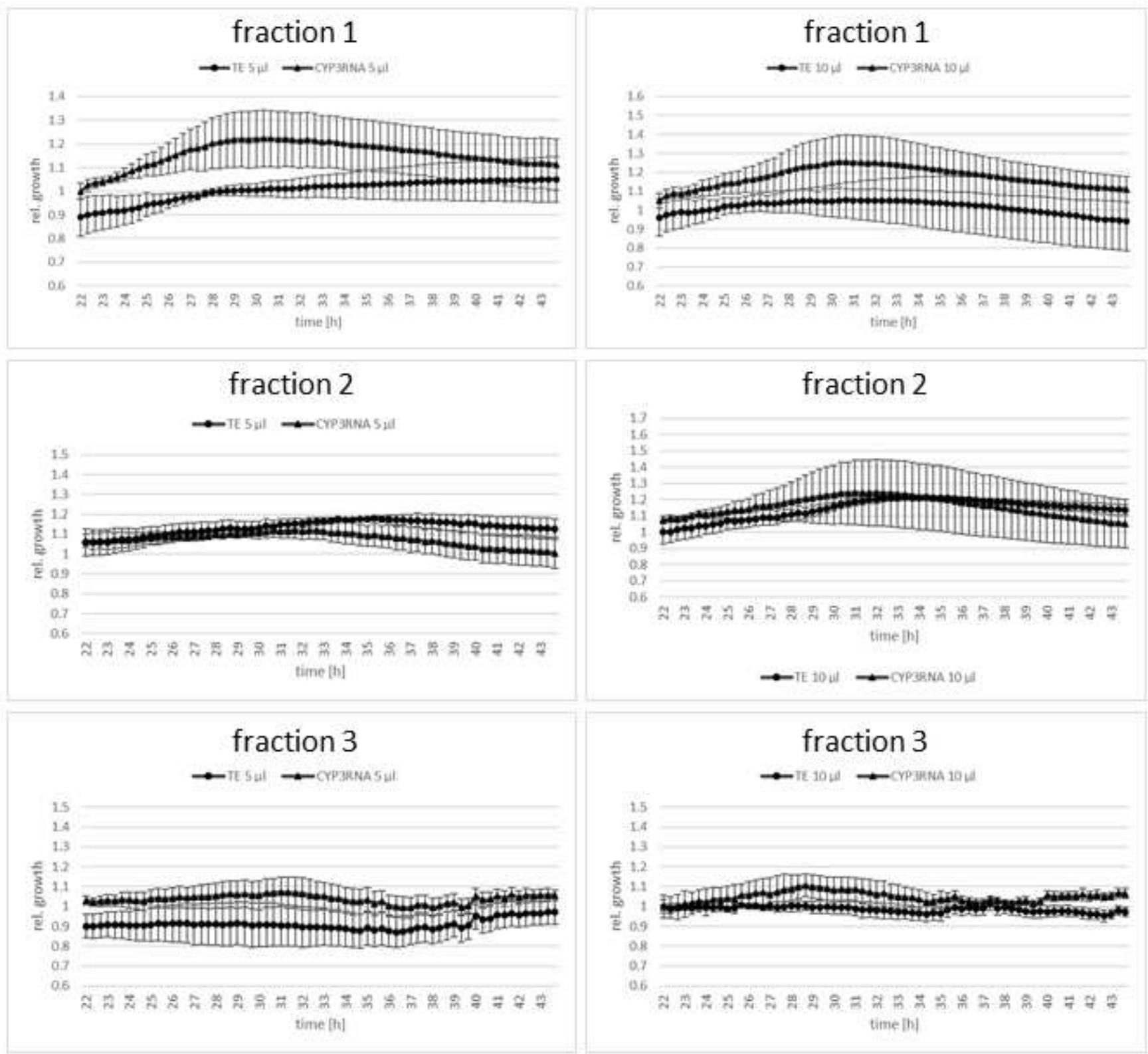

\section{Figure 5}

The relative growth was calculated from co-culture assays with differently treated barley EVs compared with the EV-free cultivation using the enzymes and detergents used for EV treatment. Control (TE: TrisEDTA): circle; CYP3RNA: triangle. 


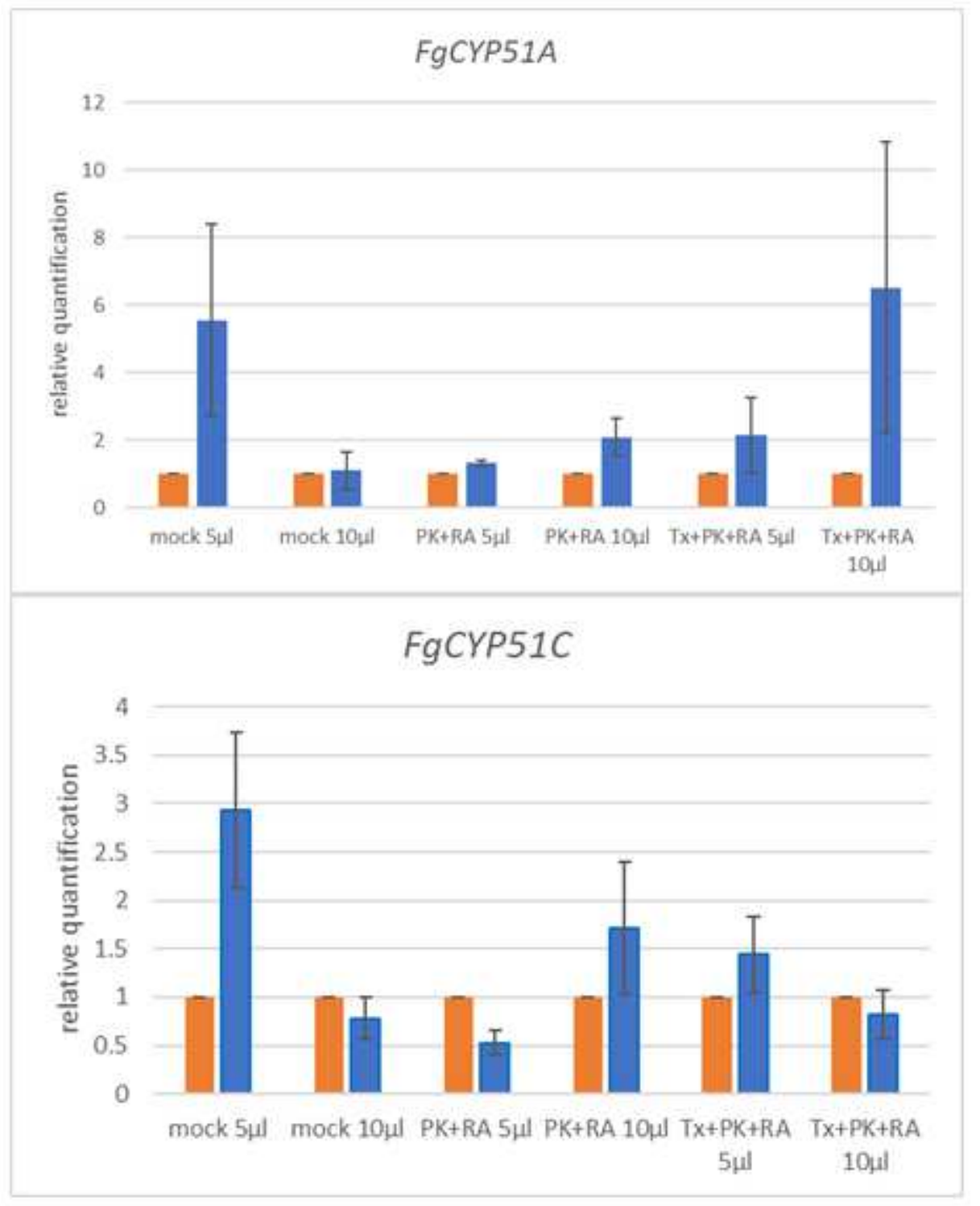

\section{Figure 6}

42 hpi of EV-Fg co-cultivation, Fg suspension was harvested and technical triplicates for each well were combined before RNA isolation. Transcriptional analyses were performed and FgCYP51A and FgCYP51C expression was calculated with the Delta Delta CT method using the elongation factor $1 \mathrm{a}$ as the reference gene. Relative quantification was determined for the equivalently co-incubated Fg cultures with EVs derived from control-sprayed and equally treated EV fractions. 